\title{
Kaposi Sarcoma in A HIV Positive Nigerian Child: A Case Report.
}

\author{
Francis Korie, ${ }^{1}$ Emeka Onuh, ${ }^{1}$ Chimaobi Iwegbulam, ${ }^{1}$ Martin Nnoli, ${ }^{2}$ \\ Charles Nwafor ${ }^{2}$ \\ ${ }^{I}$ Department of Paediatrics, Federal Medical Centre, Umuahia, Nigeria, \\ ${ }^{2}$ Department of Histopathology, Federal Medical Centre, Umuahia, Nigeria.
}

\begin{abstract}
AIDS-related Kaposi sarcoma is a malignancy thought to arise from endothelial cells. It is the commonest malignancy seen in HIV infected patients. In the USA, it was the initial presentation in $15 \%$ of homosexual men. In Africa, it is common in heterosexual adults. Compared to other regions, it is reported to be higher in East Africa. Positivity to human herpes virus 8(HHV8) is known to predate the development of Kaposi sarcoma by 5-10 years. This interval is further shortened in patients who have HIV infection prior to seroconversion to HHV8 positivity. The disease usually develops in HIV infected patients with severe immunodeficiency. Kaposi's sarcoma $(K S)$ is extremely rare among children in the developed countries and was rare in the developing countries prior to the AIDS epidemic. We report here, a 6year old female who presented with severe weight loss, swellings in oropharyngeal region with significant lymphadenopathy in the neck, axillary and inguinal regions. Histopathological studies confirmed the diagnosis of Kaposi sarcoma.
\end{abstract}

Key Words: Children, HIV infection, Kaposi sarcoma, Nigeria,.

\section{Introduction}

Kaposi sarcoma (KS) is a spindle-cell tumor, thought to be derived from endothelial cell lineage. It was initially described in 1872 by a Hungarian dermatologist Moritz Kaposi. Clinical course is variable ranging from a mild mucocutaneous disease to extensive organ involvement.

Aids associated KS lesions have no site of predilection but involvement of lymph nodes, gut and wide dissemination tend to occur early in the course. Most of these patients now succumb to opportunistic infections. About $1 / 3$ of these patients develop secondary lymphoma, most often lymphoma.

Based on clinical presentation, it can be primarily categorized into four.

1. Epidemic AIDS-related KS- This is the commonest form of presentation especially in patients with advanced HIV infection. It is common in heterosexual adults especially of African descent. Incidence is also highest where access to highly active anti-retroviral therapy (HAART) is limited. Visceral involvement is common and it represents the clinical aggressive form of KS. It occurs less commonly in children.

2. Immunocompromised KS- Occurs following solid organ transplant or in patients receiving immunosuppressive therapy. Visceral involvement is common and it also runs an aggressive course.

3. Classic (sporadic) KS-occurs primarily in elderly men of Mediterranean and eastern European background. The male: female ratio is about 15:1. Age of onset is between 50-70years. It has a protracted and indolent course. Venous stasis and lymphedema are common complications.

4. Endemic/African KS- can occur in men, women or children in Africa who are HIV negative. It was common before the AIDS epidemic. It may run an indolent or an aggressive course.

Macroscopically, cutaneous lesions may occur at any location but lower extremities, head and neck regions are typical. The lesions may be papular, nodular or plaque-like. It may assume a red, pink, brown or violaceous color and are typically non-pruritic. Mucous membrane involvement is common (palate, gingival, etc). Ulcerated or bulky tumors may interfere with speech or mastication.

Histologically, there is proliferation of spindle cells, prominent slit-like vascular spaces and extravasated red blood cells.

\section{Case Report}

N.U is a 6yr old female who presented with severe weight loss, with generalized, non tender swellings involving the cervical, axillary and inguinal groups of lymph nodes of 9 months, bilateral leg swelling and poor appetite of 1 week, all prior to presentation. There was facial puffiness, but no obvious urinary symptoms.

At onset of symptoms, patient presented to a health facility where a diagnosis of HIV stage 2 was made 4 months prior to presentation. She was subsequently started on HAART two months later. She was then referred to our centre for further evaluation and management. 
Past medical history revealed that there was blood transfusion at 6 months of life on account of anaemia. Blood was commercially procured. She is the 4 th child in a family with 4 children. Retroviral status of parents and siblings are not known.

Examination revealed an afebrile but pale child, with bilateral pitting leg oedema (grade3) and significant, generalized (cervical, axillary, inguinal) lymphadenopathy. The largest cervical nodes measured $6 \times 10 \mathrm{~cm}$. Some of the nodes were matted together. Initial ENT examination revealed masses in the orophapharynx with a bilaterally enlarged tonsil and partially occluded nasal cavities with mucopurulent discharge.

An initial diagnosis of Tuberculous Adenitis, R/O Non-Hodgkin's Lymphoma, in an RVD patient was made. The following investigations were requested: serum electrolytes, urea and creatinine, chest X-ray, full blood count, lymph node biopsy for histology and urinalysis. CXR showed right hilar opacity.

While results of histology was being awaited, progressive noisy breathing with increasing difficulty at swallowing and speech were noted. Further ENT examination revealed swollen soft palate. Both the tongue and soft palate had brownish nodules with both tonsils being enlarged and a partial occlusion of the buccal cavity. Macroscopic examination of the axillary lymph node specimen showed a nodular encapsulated grayish tissue with islands of yellowish fatty tissue attached to the capsule, measuring $3.8 \times 2 \times 1.7 \mathrm{~cm}$. Cut section shows light grayish to brown surfaces.

Histological section showed a malignant neoplastic lesion composed of numerous bundles of spindleshaped cell, dispersed in a herring bone pattern. In between spindle cells are slit like spaces containing extravasated red blood cells. The above features were in keeping with Kaposi Sarcoma. Patient was subsequently referred to another tertiary health facility for chemotherapy, radiotherapy, and further management.

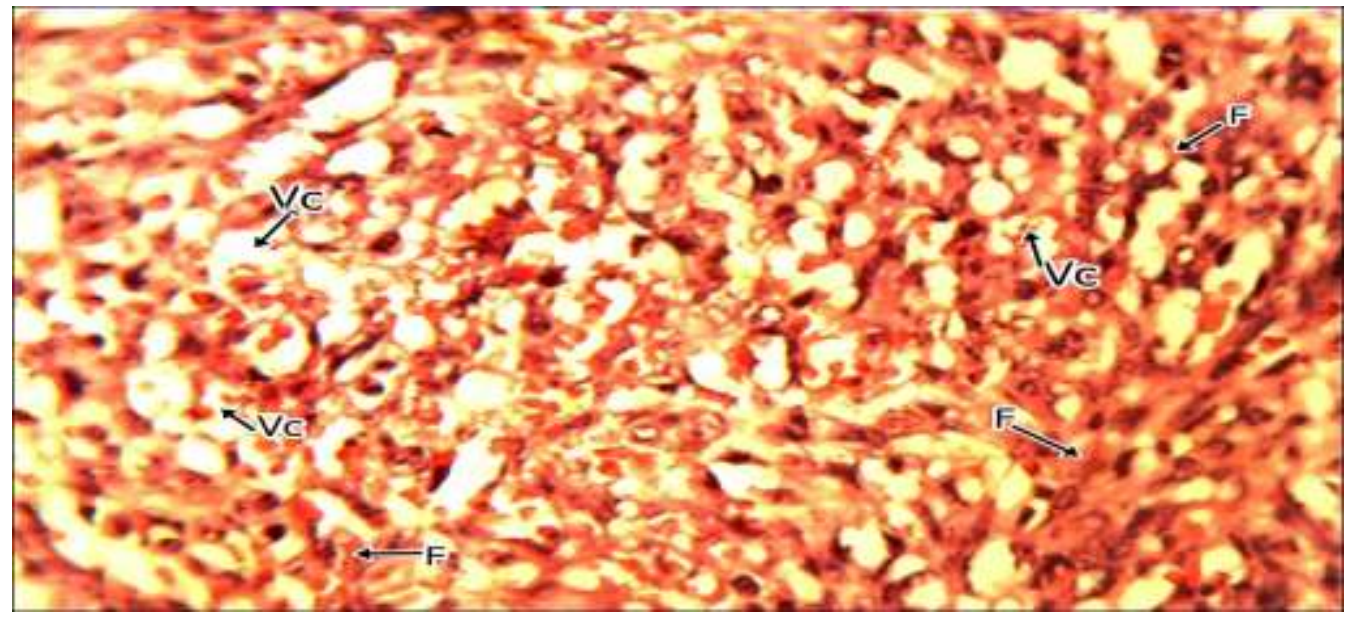

SLIDE A

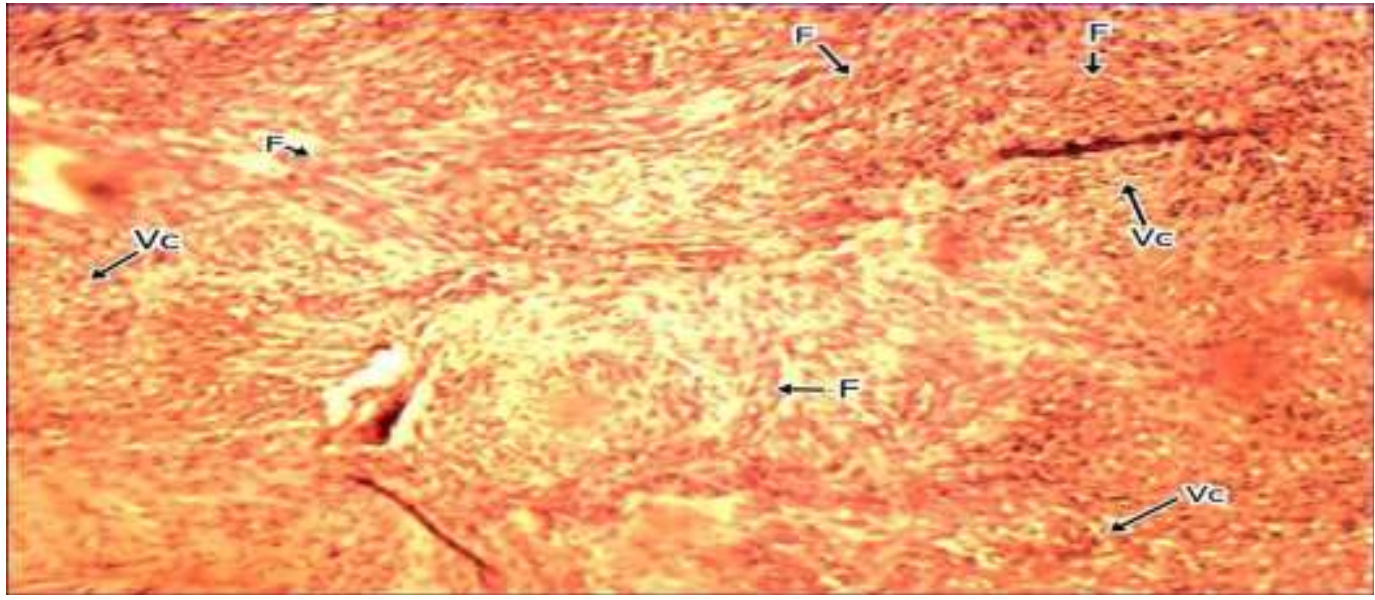

SLIDE B

A and B show histology of tumour cells. Haematoxylin and eosin sections (x100) showing vascular channels (vc) and fibroblasts (F) 


\section{Discussion}

Kaposi Sarcoma (KS) is a spindle cell tumor thought to arise from endothelial cells. Co-infection with HIV and HIV- associated herpes virus (HHV8), iatrogenic immunosuppression (use of corticosteroids) and elevated degree of expression of cytokines and growth factors (TNF- $\alpha, \mathrm{IL}-6, \beta \mathrm{FGP}$ etc), have all been implicated in its aetiology. Most convincing evidence for viral etiology was proposed following the discovery of DNA sequences of HHV8 in KS lesions in almost all AIDS patients. The pathophysiology for development of KS following HHV8 infection is not certain, but it has been postulated that HHV8 encodes several genes that have capacity to stimulate angiogenesis by inducing angiogenic transformation of HHV8 infected cells or by production of inflammatory cytokines, growth factors that promote angiogenesis.

Recent data in a study of 98 patients with KS with primary cutaneous disease analyzed by molecular diagnostic techniques comparing HHV8 DNA of the tumors showed that nearly $80 \%$ of the tumors arose independently from multiple cells. Previous studies had suggested that KS originate from a single clone.

AIDS related KS carries a variable clinical course. Lesions may involve the skin, oral mucosa, lymph nodes or visceral organs. Most patients present with cutaneous disease. The oral cavity will be affected in up to $71 \%$ of subjects with AIDS related KS. Neoplasms of the oral cavity are manifestation of KS in children infected with HIV/AIDS. The growth in the oral cavity of our patient could be an example of the oral manifestation of AIDS-related Kaposi sarcoma. The tendency to develop oral KS (and oral leukoplakia) is known to increase with age due to longer exposure to HHV8 and EBV. Visceral disease may occasionally precede cutaneous manifestation. This was the case in our patient who developed extensive oropharyngeal lesions without cutaneous manifestations. Cutaneous lesions are non pruritic and may be discrete or confluent.

The oedema often seen is typically manifested by lower extremity and facial involvement, as was seen in our patient. This is thought to occur as a result of lymphatic obstruction. Gastrointestinal disease is usually an indicator of advanced HIV infection. Pulmonary involvement may manifest as cough, dyspnoea or chest pain. It may also be a non-specific asymptomatic radiographic finding including reticulonodular or interstitial infiltrates, pleural effusion or hilar lymphadenopathy. This non specificity, in addition to the high burden of tuberculosis and other opportunistic infections, makes KS of the lungs to be highly misdiagnosed.

Lymph node involvement may be the only presenting feature. The brain is typically spared.

AIDS- related KS generally occurs in young to middle aged adults aged 20-54yrs. However, the child in this report was much younger. Other reports of KS in the Paediatric age group were also in older children who had had long standing disease.

Children known to have had prior blood transfusions probably resulting in HIV infection have also been reported to have KS. These children did not have features that might distinguish them from those who acquired HIV infection from their mothers.

A good histopathology examination is needed to distinguish this disease from bacillary angiomatosis- a disease of the immunocompromised also presenting with cutaneous nodules. Punch biopsy of skin, lymph node or an endoscopic pleural or trans- bronchial biopsy is necessary to establish the diagnosis of KS.

In the absence of a staging system, the AIDS clinical trial group (ACTG) has classified KS into:

1. Good risk: tumor confined to skin, lymph node or minimal non-nodular oral disease; CD4 of $>150 / \mathrm{mm}^{3}$, without history of history of opportunistic infections ,thrush, unexplained fever or unintentional weight loss of $>10 \%$.

2. Poor risk: tumor associated with oedema, ulceration or extensive oral Kaposi sarcoma or gastrointestinal KS; CD4 count of $<150 / \mathrm{mm}^{3}$, with history of opportunistic infection, thrush, unexplained fever or other HIV related illness, eg lymphoma.

Primary prevention is by prophylaxis against exposure to aetiologic agents HIV and HHV8. In secondary prevention, the use of HAART decreases the risk of KS in patients already infected with HIV and may be equally efficacious in the treatment of KS. This response to HAART is between $20-80 \%$ and is dependent on the stage of the disease.

Beyond HAART, the choice of therapy depends on the extent of the disease, presence or nature of symptom, or rate of disease progression. Radiation is the most widely used and effective therapy. Other treatment options include surgical excision, intralesional therapy with vinca alkaloids, cryotherapy and laser photocoagulation.

\section{Conclusion}

AIDS- related KS occurs in Nigerian children, though this is uncommon when compared to other African subregions. Survival has been shown to correlate significantly with prompt antiretroviral therapy. Punch biopsy of muco-cutaneous lesions (and indeed lesions at other accessible sites) is necessary to make a diagnosis. Screening for HHV8 would be necessary in subsequent studies, as this could not be done due to lack of reagents. Clinical photographs of the patient could not be obtained because mother did not give consent . Patients with AIDS-related KS need to be diagnosed and treated with HAART and cytotoxic drugs promptly to reduce morbidity and mortality. 


\section{References}

[1] Oladokun R, Kolude R, Ogun G, Brown B, Osunusi K. Kaposi Sarcoma in HIV Positive Nigerian Children: A Case Series. World J AIDS(1):2011;63-9.

[2] Asuquo M, Umana A, Otei O, Bassey I, Ebughe, G. Kaposi Sarcoma in Calabar, Southern Nigeria. OMJ; (24): 2009; 33.

[3] Cairncross LL, Davidson A, Millar AJ, Pillay K. Kaposi sarcoma in children with HIV: a clinical series from Red Cross Children's Hospital. J Pediatr Surg. 2009; (44):373-6.

[4] Mbulaiteye SM, Ziegler JL, Katongole-Mbidde E, Tindyebwa D, Marrum L, Newton R, et al.

[5] Risk Factors for Childhood Kaposi's Sarcoma in Uganda: A Case Control Study: 7.

[6] J Acq Immune Deficiency Syndr \& Human Retrovirology. 1997; (14):18.

[7] Vaz P, Macassa E, Jani I, Thome B, Mahagaja E, Madede T, et al. Treatment of Kaposi Sarcoma in HIV-1-infected Mozambican children with antiretrovirals and chemotherapy. A paper presented at 2nd International Workshop on HIV Pediatrics, Vienna, Austria, 16-17 July, 2010.

[8] Lager I, Altini M, Coleman H, Ali H. Oral kaposi’s Sarcoma: A Clinicopathol study from South Africa.

[9] Oral Surg, Oral Med, oral Path, Oral Radiol, and Endodont. 2003;(96): 701-10.

[10] Agarwal AK, Zamzachin G, Mohen Y, Singh MY, Singh N. AIDS-related Kaposi's Sarcoma-Like Lesion.

[11] Indian J of Dermatol, 1996; 62):129-31.

[12] Duprez R, Lacoste V, Briere J, Coupie P, Frances C, Sainte-Marie D, et al. Evidence for a multiclonal origin of multicentric advanced lesions of Kaposi Sarcoma. J Nat Cancer Inst. 2007; (99):1086-94.

[13] Kline MW. Oral manifestations of Paediatric Human Immunodeficiency Virus: A Review of the Literature. Paediatr. 1996;(97):380-8.

[14] Mosun A, Aboobaker J, Shaik F. Kaposi's Sarcoma in Sub-Saharan Africa: A Current Perspective. Current Opinion in Infect Dis. 2010; (23):119-23

[15] Okunseri C, Badner V, Wiznia A, Rosemberg M. Prevalence of Oral Lesions and percent CD4+ T- Lymphocytes in HIV Infected Children on Antiretroviral Therapy. AIDS Patient Care and STDS.2003;(17):5-11.

[16] Ziegler JL, Katongole-Mbidde E. Kaposi's Sarcoma in Childhood: An Analysis of 100 Cases from Uganda and Relationship to HIV Infection. Int J Cancer.1996;(65):200-3.

[17] Stefan DC, Stones DK, Wainwright L, Newton R. Kaposi's Sarcoma in South African Children. Paediatr Blood Cancer. 2011; (56):392-6.

[18] Cattelan AM, Calabro ML, DeRossi A, et al. Long-term clinical outcome of AIDS-related Kaposi's Sarcoma during highly active antiretroviral therapy. Internat J Oncol. 2005;(27): 779-85.

[19] Nobler MP, Leddy ME, Huh SH. The impact of palliative irradiation on the management of patients with acquired immune deficiency syndrome. J Clinic Oncol. 1987;(5):107-12.

[20] Kemeny L, Gyulai R, Kiss M, Nagy F, Dobozy A. Kaposi's sarcoma-associated herpes virus/human herpes virus 8: a new virus in human pathology. J Am Acad Dermatol. 1997;(37):107. 